\title{
Avaliação da qualidade nos serviços públicos de saúde com base na percepção dos usuários e dos profissionais
}

\section{Quality evaluation in health services based on perception of users and professional}

Luciana Fernandes Volpato Martins* Marcelo de Castro Meneghim ${ }^{* *}$

Luiz Cândido Martins ${ }^{* * *}$

Antonio Carlos Pereira ${ }^{* * *}$

\section{Resumo}

O SUS assegurou a universalização do direito a serviços de saúde eficientes, tornando a busca pela qualidade um desafio. Para isso, a percepção dos usuários e dos profissionais é importante para avaliar a qualidade dos serviços, seja na atenção básica, na média ou na alta complexidade. Este estudo apresenta a hipótese de uma limitação nas pesquisas sobre a qualidade dos serviços públicos de saúde no Brasil, pois essas enfatizam a percepção dos usuários, deixando a dos profissionais em um plano inferior de relevância. Objetivo: o objetivo deste estudo foi verificar a percepção dos usuários e profissionais na avaliação da qualidade nos serviços públicos de saúde, através de uma revisão sistemática. Materiais e método: foi realizada uma busca nas bases bibliográficas Medline, Lilacs e Scielo, no período de 1990 a 2013, utilizando as palavras-chave: qualidade em serviços de saúde, satisfação do usuário, satisfação dos consumidores, satisfação no emprego e trabalhadores de saúde. Resultados: os resultados mostraram que a atenção básica foi objeto de aplicação da análise na maioria dos estudos, o método de pesquisa quantitativo foi o mais utilizado e a percepção dos usuários, a mais aferida. Conclusão: concluiu-se que na atenção básica, a boa relação profissional-usuário, o número adequado de profissionais e as boas condições na estrutura física foram características de qualidade comuns aos usuários e profissionais. A média complexidade apresentou o item facilidade para agendar consulta comum à percepção dos usuários e dos profissionais. A boa relação profissional-usuário foi comum à percepção dos usuários e de profissionais no que diz respeito à alta complexidade.

Palavras-chave: Qualidade da assistência em saúde. Epidemiologia. SUS.

\section{Introdução}

A criação do Sistema Único de Saúde (SUS), em 1988, assegurou a todo cidadão o direito a serviços de saúde eficientes e de qualidade. Ao se tornar um direito, a saúde teve seu acesso ampliado à população, mas a eficiência e a qualidade dos serviços prestados são questionadas constantemente, mostrando sua ineficácia e ineficiência através das imensas filas e dos atendimentos em macas espalhadas pelos corredores $^{1,3}$.

Diante das dificuldades da saúde pública, o Governo Federal desenvolveu o Programa Mais Médicos, em 2013, com a intenção de ampliar o número de médicos na atenção básica das periferias de grandes cidades e municípios do interior do país, onde há escassez e ausência de profissionais. Os

\footnotetext{
Doutoranda em Saúde Pública no Departamento de Odontologia Social na Faculdade de Odontologia de Piracicaba - FOP/Unicamp, Piracicaba/SP, Brasil.

- Professor Doutor junto ao Departamento de Odontologia Social na Faculdade de Odontologia de Piracicaba - FOP/Unicamp, Piracicaba/SP, Brasil.

** Professor Doutor, Programa de Pós-Graduação em Educação na Universidade Metodista de Piracicaba - Unimep, Piracicaba/SP, Brasil.

*.* Professor Doutor junto ao Departamento de Odontologia Social na Faculdade de Odontologia de Piracicaba - FOP/Unicamp, Piracicaba/ SP, Brasil.
} 
médicos com diploma no Brasil tiveram prioridade e as vagas remanescentes foram oferecidas, primeiramente aos brasileiros graduados no exterior e, em seguida, aos estrangeiros. Antes de iniciar as atividades, os médicos estrangeiros foram avaliados e supervisionados por universidades federais, de todas as regiões do País que se inscreveram no programa, e começaram a atuar com autorização profissional provisória, restrita à atenção básica e às regiões nas quais foram alocados pelo programa.

Trava-se, atualmente, um debate de importante proporção e alcance político entre os Conselhos de Medicina (Regional e Federal) e o Governo, pelo fato de os médicos estrangeiros já presentes no país terem sido isentos do exame Revalida. Muitos Conselhos de Medicina estão negando aos médicos estrangeiros credenciais que os habilitem ao desenvolvimento de suas funções. Não se pretende, neste estudo, o juízo de mérito, senão a constatação do fato e a evidência das dificuldades dos serviços públicos de saúde no Brasil, pela falta de estrutura física, pela falta de profissionais e pelas condições dignas de trabalho para esses.

Nos serviços públicos de saúde, a busca pela qualidade se faz necessária devido às profundas desigualdades sociais, às péssimas condições de vida e de saúde da população, à má alocação dos recursos, à ineficiência, aos custos crescentes e à desigualdade nas condições de acesso dos usuários ${ }^{4,5}$.

A avaliação, compreendida como parte do processo de gestão, objetiva maior eficiência e efetividade dos serviços, considerando os usuários e os profissionais através de suas percepções e necessidades ${ }^{2,6}$.

Avaliar a satisfação dos usuários é fundamental para a gestão dos serviços, pois pode indicar decisões estratégicas e operacionais que venham a influenciar na qualidade dos serviços prestados pelo sistema de saúde. Essas avaliações expressam uma resposta efetiva de que o cuidado possui certos atributos que podem ser avaliados pelos usuários ${ }^{7-9}$.

A qualidade dos serviços de saúde depende da responsabilidade do profissional e da sua experiência, do seu compromisso com a organização, com os usuários e com as atividades que desenvolve ${ }^{2,10}$. Diante disso, é necessário averiguar as necessidades e as expectativas dos profissionais, sua satisfação com o trabalho e sua visão dos usuários, uma vez que, na organização de serviços, há uma relação de troca entre os sujeitos e esse relacionamento refletirá na qualidade do serviço ${ }^{11,12}$.

Assim, a percepção dos usuários sobre os serviços de saúde permite avaliar sua relação com o profissional, o trato humanitário durante o atendimento $^{13}$ e a satisfação do atendimento ${ }^{14,15}$. Por sua vez, a percepção do profissional permite diagnosticar as características do serviço, segundo a ótica dos próprios funcionários em relação aos seus aspectos administrativos e no que diz respeito à relação profissional-usuário ${ }^{2,10}$.

Conhecer a percepção dos usuários e dos profissionais, no que se refere à qualidade, pode ser um primeiro passo para o desenvolvimento de ações que conduzirão à melhoria do serviço de saúde, tanto por parte do gestor do sistema, que precisa conhecer a percepção de ambos para melhor direcionar suas estratégias e ações, como por parte dos próprios profissionais que, entendendo a percepção da qualidade sob a perspectiva de seus usuários, estarão mais preparados para atender suas expectativas ${ }^{2,16}$.

Neste estudo, discute-se a hipótese de que há uma limitação nas pesquisas sobre a qualidade nos serviços públicos de saúde no Brasil, uma vez que apresentam forte ênfase na perspectiva dos usuários, enquanto indicador de qualidade, deixando a perspectiva dos profissionais, que também é um indicador de qualidade, relegado a um plano de relevância inferior.

O objetivo deste estudo foi verificar a percepção dos usuários e dos profissionais na avaliação da qualidade nos serviços públicos de saúde, por meio de uma revisão sistemática.

\section{Materiais e método}

Para a elaboração da revisão sistemática, foi realizada uma busca ativa de informações nas bases bibliográficas Medline, Lilacs e Scielo no período compreendido entre 1990 e 2013. Considerou-se a data de 1990 para iniciar o levantamento de artigos científicos, pois os estudos sobre a percepção da qualidade nos serviços de saúde foram mais frequentes a partir desse ano.

As buscas eletrônicas foram realizadas utilizando-se as seguintes palavras-chave: qualidade em serviços de saúde, satisfação do usuário, satisfação dos consumidores, satisfação no emprego e trabalhadores de saúde.

Foram incluídos artigos que abordam resultados de pesquisas com a população brasileira nos serviços públicos de saúde da atenção básica, de média e alta complexidade e que abordavam a percepção dos usuários e/ou dos profissionais. Estudos internacionais, revisões sistemáticas e de literatura, teses, dissertações e pesquisas populacionais foram excluídos desta pesquisa.

Dos artigos selecionados, foram retiradas informações sobre as características do artigo (autores e ano de publicação), o local da pesquisa, o tipo de assistência em saúde, o método da pesquisa, a percepção dos usuários e dos profissionais sobre os serviços de saúde.

\section{Resultados}

Foram identificados 195 artigos nas bases Medline, Lilacs e Scielo, entretanto, após a leitura, 165 artigos foram excluídos, pois 54 estavam repetidos e 111 não correspondiam aos critérios de inclusão. Assim, foram para análise 30 artigos, apresentados no Quadro 1, em que estão descritas as principais características sobre a percepção dos usuários e/ou dos profissionais referente à qualidade dos serviços públicos de saúde. 
Quadro 1 - Principais características dos estudos brasileiros sobre percepção dos usuários e dos profissionais.

\begin{tabular}{|c|c|c|c|c|c|}
\hline $\begin{array}{l}\text { Autores, } \\
\text { ano publicação }\end{array}$ & Local & $\begin{array}{l}\text { Tipo da } \\
\text { assistência }\end{array}$ & $\begin{array}{l}\text { Método da } \\
\text { pesquisa }\end{array}$ & Percepção dos usuários & Percepção dos profissionais \\
\hline Halal et al. ${ }^{17}$ (1994) & Pelotas/RS & Atenção Básica & Quantitativo & $\begin{array}{l}\text { Facilidade no agendamento de consulta no serviço e nos servi- } \\
\text { ços especializados, resolução do problema. }\end{array}$ & $\begin{array}{l}\text { Boa relação profissional-usuário, } \\
\text { educação continuada. }\end{array}$ \\
\hline $\begin{array}{l}\text { Franco e Campos }{ }^{18} \\
\text { (1998) }\end{array}$ & Campinas/SP & $\begin{array}{c}\text { Média } \\
\text { complexidade }\end{array}$ & Quantitativo & $\begin{array}{l}\text { Boa relação profissional-usuário, tempo de espera, intervalo } \\
\text { para retornos. }\end{array}$ & \\
\hline Kloetzel et al. ${ }^{19}(1998)$ & Areal/RJ & Atenção Básica & Quantitativo & $\begin{array}{l}\text { Facilidade no agendamento de consulta, tempo de espera, } \\
\text { cordialidade no atendimento, confiança na equipe. }\end{array}$ & \\
\hline Ramos e Lima $^{15}(2003)$ & Porto Alegre/RS & Atenção Básica & Qualitativo & Boa relação profissional-usuário, tempo de espera. & \\
\hline Kovacs et al. ${ }^{20}(2005)$ & Recife/PE & $\begin{array}{c}\text { Média } \\
\text { complexidade }\end{array}$ & Quantitativo & $\begin{array}{l}\text { Tempo de espera, resolução do problema, acessibilidade ge- } \\
\text { ográfica. }\end{array}$ & \\
\hline $\begin{array}{l}\text { Silveira, Hayashi } \\
\text { e Scarpi }{ }^{21}(2005)\end{array}$ & São Paulo/SP & $\begin{array}{c}\text { Média } \\
\text { complexidade }\end{array}$ & $\begin{array}{l}\text { Quantitativo e } \\
\text { Qualitativo }\end{array}$ & $\begin{array}{l}\text { Cordialidade no atendimento, tempo de espera, resolução do } \\
\text { problema. }\end{array}$ & \\
\hline Oliveira e Spiri"1 (2006) & Conchas/SP & Atenção Básica & Qualitativo & & $\begin{array}{l}\text { Educação continuada, boa rela- } \\
\text { ção profissional-usuário, trabalho } \\
\text { em equipe. }\end{array}$ \\
\hline Ferri et al. ${ }^{22}(2007)$ & Interior/SP & Atenção Básica & Qualitativo & $\begin{array}{l}\text { Manutenção do local, tempo de espera, facilidade no agen- } \\
\text { damento de consulta, cordialidade no atendimento. }\end{array}$ & \\
\hline Jorge et al. ${ }^{23}(2007)$ & $\begin{array}{l}\text { Fortaleza, Quixadá, } \\
\text { Aracati, Cascavel e } \\
\text { Canindé/CE }\end{array}$ & Atenção Básica & Quantitativo & $\begin{array}{l}\text { Cordialidade no atendimento, boa relação profissional- } \\
\text {-usuário. }\end{array}$ & \\
\hline Lima et al. ${ }^{24}$ (2007) & Porto Alegre/RS & Atenção Básica & Qualitativo & $\begin{array}{l}\text { Tempo de espera, facilidade no agendamento de consulta, } \\
\text { cordialidade no atendimento. }\end{array}$ & \\
\hline Paiva e Gomes ${ }^{25}$ (2007) & Interior/SP & Alta complexidade & Qualitativo & $\begin{array}{l}\text { Manutenção do local, capacitação profissional, boa relação } \\
\text { profissional-usuário, profissionais sobrecarregados. }\end{array}$ & \\
\hline $\begin{array}{l}\text { Santos, Meneghim e } \\
\text { Pereira }^{26}(2007)\end{array}$ & $\begin{array}{l}\text { Campos dos } \\
\text { Goytacazes/RJ }\end{array}$ & $\begin{array}{l}\text { Média } \\
\text { complexidade }\end{array}$ & Quantitativo & $\begin{array}{l}\text { Facilidade no agendamento de consultas, boa relação pro- } \\
\text { fissional-usuário. }\end{array}$ & $\begin{array}{l}\text { Organização do serviço, facili- } \\
\text { dade no agendamento de con- } \\
\text { sultas, demanda de usuários. }\end{array}$ \\
\hline $\begin{array}{l}\text { De Marco et al. } .^{27} \\
\text { (2008) }\end{array}$ & São Paulo/SP & Alta complexidade & Quantitativo & & $\begin{array}{l}\text { Relação entre os profissionais, } \\
\text { serviços oferecidos, impacto } \\
\text { emocional. }\end{array}$ \\
\hline Moura et al. ${ }^{28}(2008)$ & Fortaleza/CE & Atenção Básica & Quantitativo & $\begin{array}{l}\text { Cordialidade no atendimento, organização da recepção, ma- } \\
\text { nutenção do local. }\end{array}$ & \\
\hline Mendes et al..$^{29}(2009)$ & Recife/PE & Alta complexidade & Quantitativo & $\begin{array}{l}\text { Cordialidade e privacidade no atendimento, tempo de espe- } \\
\text { ra, falta de profissionais, manutenção do local. }\end{array}$ & \\
\hline Souza et al. ${ }^{30}(2009)$ & Fortaleza/CE & Alta complexidade & Quantitativo & Tempo de espera, cordialidade no atendimento. & \\
\hline $\begin{array}{l}\text { Benazzi, Figueiredo e } \\
\text { Bassani }^{31}(2010)\end{array}$ & Canoas/RS & $\begin{array}{c}\text { Média } \\
\text { complexidade }\end{array}$ & Quantitativo & $\begin{array}{l}\text { Cordialidade no atendimento, tempo de espera, falta de pro- } \\
\text { fissionais, consultas rápidas. }\end{array}$ & \\
\hline $\begin{array}{l}\text { Lima, Cabral e } \\
\text { Vasconcelos }{ }^{32}(2010)\end{array}$ & Recife/PE & $\begin{array}{c}\text { Média } \\
\text { complexidade }\end{array}$ & Quantitativo & $\begin{array}{l}\text { Facilidade no agendamento de consulta, tempo de espera, } \\
\text { boa relação profissional-usuário. }\end{array}$ & \\
\hline Medeiros et al..$^{33}(2010)$ & $\begin{array}{l}\text { São Gonçalo do } \\
\text { Amarante/RN }\end{array}$ & Atenção Básica & $\begin{array}{l}\text { Quantitativo e } \\
\text { Qualitativo }\end{array}$ & $\begin{array}{l}\text { Facilidade no agendamento de consulta e no encaminha- } \\
\text { mento aos serviços especializados, resolução dos problemas, } \\
\text { boa relação profissional-usuário. }\end{array}$ & \\
\hline $\begin{array}{l}\text { Nery, Marcelo e } \\
\operatorname{Dantas}^{34}(2010)\end{array}$ & Goiânia/GO & Atenção Básica & Qualitativo & $\begin{array}{l}\text { Facilidade no agendamento de consulta, tempo de espera, } \\
\text { estrutura do local. }\end{array}$ & \\
\hline Schwartz et al..$^{35}(2010)$ & Vitória/ES & Atenção Básica & Qualitativo & $\begin{array}{l}\text { Facilidade no agendamento de consulta no serviço e nos servi- } \\
\text { ços especializados, rotatividade profissional, tempo de espera. }\end{array}$ & \\
\hline Souza e Soares ${ }^{36}(2010)$ & Rio de Janeiro/RJ & $\begin{array}{c}\text { Média } \\
\text { complexidade }\end{array}$ & Qualitativo & & $\begin{array}{l}\text { Processo de trabalho, diálogo em } \\
\text { equipe. }\end{array}$ \\
\hline Volpato et al. ${ }^{2}(2010)$ & Piracicaba/SP & Atenção Básica & Qualitativo & $\begin{array}{l}\text { Facilidade para agendar consultas no serviço e nos serviços es- } \\
\text { pecializados, cordialidade no atendimento, tempo de espera. }\end{array}$ & $\begin{array}{l}\text { Educação continuada, motiva- } \\
\text { ção profissional, incentivo finan- } \\
\text { ceiro, rotatividade profissional. }\end{array}$ \\
\hline $\begin{array}{l}\text { Gonçalves et al. }{ }^{37} \\
(2011)\end{array}$ & Campo Maior/PI & $\begin{array}{l}\text { Média } \\
\text { complexidade }\end{array}$ & Quantitativo & $\begin{array}{l}\text { Acesso ao serviço, tempo de espera, disponibilidade de apa- } \\
\text { relhos, esclarecimento sobre procedimentos, boa relação } \\
\text { profissional-usuário. }\end{array}$ & \\
\hline Ricci et al..$^{38}(2011)$ & São Carlos/SP & Alta complexidade & Quantitativo & $\begin{array}{l}\text { Tempo de espera, manutenção do local, boa relação profis- } \\
\text { sional-usuário, resolução dos problemas. }\end{array}$ & \\
\hline $\begin{array}{l}\text { Rosa, Pelegrini e } \\
\operatorname{Lima}^{39}(2011)\end{array}$ & Porto Alegre/RS & Atenção Básica & $\begin{array}{l}\text { Quantitativo e } \\
\text { Qualitativo }\end{array}$ & $\begin{array}{l}\text { Facilidade no encaminhamento a serviços especializados, } \\
\text { resolução dos problemas. }\end{array}$ & \\
\hline $\begin{array}{l}\text { Serapioni e Silva }{ }^{40} \\
\text { (2011) }\end{array}$ & Ceará & Atenção Básica & $\begin{array}{l}\text { Quantitativo e } \\
\text { Qualitativo }\end{array}$ & $\begin{array}{l}\text { Boa relação profissional-usuário, cordialidade no atendimen- } \\
\text { to, facilidade no agendamento de consulta, tempo de espera. }\end{array}$ & $\begin{array}{l}\text { Boa relação profissional-usuá- } \\
\text { rio, manutenção do local, edu- } \\
\text { cação continuada, rotatividade } \\
\text { profissional. }\end{array}$ \\
\hline $\begin{array}{l}\text { Pena e Melleiro }{ }^{41} \\
(2012)\end{array}$ & Interior/SP & Alta complexidade & Quantitativo & $\begin{array}{l}\text { Tempo de espera, atendimento ágil da campainha nos leitos, } \\
\text { boa relação profissional-usuário. }\end{array}$ & \\
\hline Sousa e Coleta ${ }^{42}$ (2012) & $\begin{array}{l}\text { Uberlândia, } \\
\text { Araguari/MG }\end{array}$ & Alta complexidade & Quantitativo & & $\begin{array}{l}\text { Boa relação entre profissionais, } \\
\text { realização profissional, contato } \\
\text { com paciente, desenvolvimento } \\
\text { profissional/pessoal, reconheci- } \\
\text { mento público. }\end{array}$ \\
\hline Santiago et al. ${ }^{43}(2013)$ & Recife/PE & Atenção Básica & Quantitativo & $\begin{array}{l}\text { Tempo de espera, facilidade no agendamento de consulta no ser- } \\
\text { viço e no serviço especializado, cordialidade no atendimento. }\end{array}$ & \\
\hline
\end{tabular}


Dos 30 artigos selecionados, constatou-se que a atenção primária em saúde foi objeto de aplicação da análise em 15 estudos, a atenção secundária em oito e a terciária em sete.

As investigações envolvendo a atenção básica em saúde foram realizadas nas regiões Sudeste (5), Sul (4), Nordeste (4), Norte (1) e Centro-oeste (1). A metodologia qualitativa (7) foi a mais frequente na atenção básica, seguida da quantitativa (5) e da qualitativo-quantitativa (3). A percepção dos usuários foi observada em 11 estudos, enquanto que a dos profissionais em um e ambas as percepções em três.

Os serviços de saúde de média complexidade foi alvo de pesquisa nas regiões Sudeste (4), Nordeste (3) e Sul (1). A metodologia quantitativa foi realizada em seis estudos, o qualitativo e o qualitativo/ quantitativo em um estudo cada um. A percepção dos usuários foi abordada em seis artigos, a dos profissionais em um e a dos usuários e dos profissionais também em um artigo.

A região Sudeste foi citada em cinco estudos realizados nos serviços de saúde de alta complexidade, enquanto a região Nordeste em dois. As pesquisas quantitativas foram aplicadas em seis estudos e a qualitativa em um e, finalmente, a percepção dos usuários foi relatada em cinco estudos, enquanto a dos profissionais em dois.

Os estudos mostraram que para garantir a qualidade nos serviços de saúde da atenção básica, segundo a percepção dos usuários, é necessário: $1^{\circ}$ ) facilidade para agendar consulta no serviço de saúde; $2^{\circ}$ ) diminuir o tempo de espera; $3^{\circ}$ ) cordialidade no atendimento; $4^{\circ}$ ) facilidade para agendar consulta no serviço especializado; $5^{\circ}$ ) boa relação profissional-usuário; $6^{\circ}$ ) resolução dos problemas; $7^{\circ}$ ) boas condições na estrutura física; $8^{\circ}$ ) número adequado de profissionais. A percepção dos profissionais revelou que a qualidade do serviço de saúde depende de: $1^{\circ}$ ) educação continuada; $2^{\circ}$ ) boa relação profissional-usuário; $3^{\circ}$ ) motivação profissional; $4^{\circ}$ ) número adequado de profissionais; $5^{\circ}$ ) trabalho em equipe e $6^{\circ}$ ) boas condições na estrutura física.

Nos serviços de média complexidade, os usuários declararam a necessidade dos seguintes itens para a qualidade do serviço: $1^{\circ}$ ) diminuir o tempo de espera; $2^{\circ}$ ) boa relação profissional-usuário, $3^{\circ}$ ) cordialidade no atendimento; $4^{\circ}$ ) facilidade para agendar consulta, $5^{\circ}$ ) resolução dos problemas; $6^{\circ}$ ) número adequado de profissionais. Já os profissionais abordaram a seguinte ordem para os itens de qualidade: $1^{\circ}$ ) a organização do serviço; $2^{\circ}$ ) a facilidade para agendar consulta e $3^{\circ}$ ) o trabalho em equipe.

A garantia da qualidade aos usuários dos serviços de alta complexidade depende de: $1^{\circ}$ ) diminuição do tempo de espera; $2^{\circ}$ ) boas condições na estrutura física; $3^{\circ}$ ) da capacitação profissional; $4^{\circ}$ ) da boa relação profissional-usuário; $5^{\circ}$ ) cordialidade no atendimento e $6^{\circ}$ ) do número adequado de profissionais. Os profissionais revelaram que a qualidade do serviço depende: $1^{\circ}$ ) trabalho em equipe; $2^{\circ}$ ) motivação profissional e $3^{\circ}$ ) boa relação profissional-usuário.

\section{Discussão}

Os resultados apontaram que, predominaram estudos na região Sudeste, nos quais se buscou, com base em metodologia de pesquisa qualitativa, esclarecer a percepção dos usuários de serviços públicos de saúde da atenção básica. Nos serviços de média complexidade, prevaleceram os estudos de método quantitativo em que se investigou a percepção dos usuários da região Sudeste. No que diz respeito à alta complexidade, as pesquisas quantitativas foram as mais estudadas as quais foram realizadas preponderantemente na região Sudeste e versaram sobre a percepção dos usuários.

Mesmo sendo a região mais rica do Brasil, o Sudeste enfrenta vários problemas nos serviços públicos de saúde, segundo as percepções dos usuários e também dos profissionais. As demais regiões apresentaram problemas igualmente graves na área da saúde pública, sobretudo o Norte e o Nordeste, onde se encontram os piores indicadores de pobreza e de desigualdade social. Para amenizar os problemas de saúde nessas regiões, o Governo Federal disponibilizou, por meio do Programa Mais Médicos, cerca de $90 \%$ dos médicos cubanos que vieram ao Brasil. Discutem-se as dimensões políticas, assim como o ponto de visto técnico, buscando-se avaliar se o Programa Mais Médicos seria ou não a solução para o grave problema da saúde pública. No entanto, essa discussão transcende aos limites deste estudo, permanecendo a constatação do vácuo de qualidade do sistema público de saúde brasileiro.

Os estudos quantitativos predominaram no que diz respeito a serviços de saúde de média e de alta complexidade. $\mathrm{Na}$ maioria das vezes, os processos avaliativos contemplam a medição quantitativa e avaliam a importância, o risco e as ameaças, além de abordar probabilidades e associações estatisticamente significantes e importantes para se conhecer uma realidade ${ }^{44}$

O campo da saúde envolve aspectos biológicos, físicos, psicológicos, sociais e ambientais. Muitas vezes, há a necessidade de ponderar acerca de valores, atitudes e crenças dos grupos estudados, assim, o binômio saúde-doença traz uma carga histórica, cultural, política e ideológica que não está contida numa fórmula numérica ou num dado estatístico ${ }^{44-46}$.

A pesquisa qualitativa, geralmente, analisa o comportamento, as perspectivas e as experiências dos voluntários. Os aspectos subjetivos podem levar a resultados objetivos, desde que o pesquisador interprete os dados de acordo com o que foi transmitido pelos sujeitos pesquisados, não influenciando o tema investigado ${ }^{45,46}$.

A escolha da metodologia de estudo (qualitativa, quantitativa ou mista) tem grande importância para uma pesquisa.Assim, é necessário observar as limitações que o tipo de estudo pode acarretar nos resultados. $\mathrm{Na}$ pesquisa qualitativa algumas limitações devem ser consideradas: a subjetividade, a possibilidade de viés do observador que pode com- 
prometer a validade do estudo. Além disso,o trabalho é intenso e demorado, podendo significar um custo elevado. Já a abordagem quantitativa é restritiva, representando um retrato reducionista da complexidade social, não conseguindo captar o ponto de vista do indivíduo, por utilizar grandes amostras e afastar o pesquisador do objeto de estudo ${ }^{45,46}$.

Um bom método de pesquisa permite a construção correta dos dados, mostra-se adequado aos objetivos da investigação e oferece elementos teóricos para análise. Dessa forma, as metodologias quantitativas ou qualitativas apresentam igual valor, desde que utilizadas em função das necessidades e dos objetivos da pesquisa, mantendo o rigor científico que diferencia e dá credibilidade a qualquer estudo ${ }^{44}$.

As pesquisas sobre a percepção dos usuários prevaleceram na atenção básica, de média e alta complexidade, mas nos três níveis de assistência foram realizadas pesquisas de percepção com os usuários e com os profissionais.

$\mathrm{Na}$ atenção básica, segundo os resultados, os pontos de maior importância para a percepção dos usuários sobre a qualidade dos serviços de saúde foram: facilidade para agendar consultas no serviço e no serviço especializado, tempo de espera, cordialidade no atendimento, boa relação profissional-usuário, resolução dos problemas e boas condições na estrutura física. Para os profissionais, a qualidade do serviço depende da educação continuada, da boa relação profissional-usuário, da motivação profissional, do número adequado de profissionais e das boas condições na estrutura física.

Os usuários dos serviços de média complexidade declararam perceber os seguintes itens de qualidade: tempo de espera, boa relação profissional-usuário, cordialidade no atendimento, facilidade para agendar consultas e resolução dos problemas. Já os profissionais mencionaram a organização do serviço, a facilidade para agendar consultas e o trabalho em equipe.

A qualidade, para os usuários dos serviços de saúde de alta complexidade, depende da diminuição do tempo de espera, das boas condições na estrutura física, da capacitação profissional, da boa relação profissional-usuário, da cordialidade no atendimento e do número adequado de profissionais. Os profissionais revelaram que a qualidade do serviço depende do trabalho em equipe, da motivação profissional e da boa relação profissional-usuário.

A qualidade aferida pela percepção do usuário baseia-se no conceito que esse faz em relação ao fato de um serviço responder ou não às suas necessidades de cuidado. No entanto, é uma tarefa complexa, pois se trata de mensuração de opiniões, influenciadas por diferentes situações (aspectos culturais, sociais, pessoais e psicológicos), expectativas e antecedentes ${ }^{19,33,35}$.

A utilização de um serviço de saúde está associada às necessidades e às expectativas vinculadas a um problema de saúde. Assim, após o uso desses serviços, o usuário poderá mencionar se suas necessidades e expectativas foram atendidas. $\mathrm{O}$ nível de percepção possibilita interferir na qualidade nos diversos momentos de atendimento, desde a entrada até a saída do serviço ${ }^{2,35,47}$.

Ao avaliar a qualidade, os usuários realizam comparações entre o desempenho do serviço e suas expectativas, os resultados são bons, quando a qualidade percebida é obtida. A qualidade pode ser percebida através de aspectos tangíveis (boas condições na estrutura física) e intangíveis (cordialidade no atendimento $)^{32,48,49}$.

A percepção profissional, assim como a percepção dos usuários, é um fenômeno complexo, pois referem um estado subjetivo que varia conforme circunstâncias, pesso, podendo, ainda, variar ao longo do tempo para a mesma pessoa. A satisfação no trabalho é resultante da interação entre o profissional, suas características pessoais, seus valores, suas expectativas e o ambiente e a empresa; podendo ser observada através da qualidade do trabalho, do desempenho, da produtividade, da pontualidade, do absenteísmo e da rotatividade ${ }^{2,50,51}$.

Os profissionais nem sempre tem suas necessidades e expectativas levadas em consideração, contudo a atuação e a qualidade dos serviços de saúde dependem do profissional e de sua experiência, do seu compromisso com a organização, com os usuários e com as atividades que desenvolve. Diante disso, é necessário averiguar as necessidades e as expectativas dos profissionais, sua satisfação com o trabalho e sua visão dos usuários, uma vez que na organização de serviços há uma relação de troca entre os sujeitos e esse relacionamento refletirá na qualidade do serviço, além de proporcionar efeitos positivos ao tratamento ${ }^{2,11}$.

Ainda que as percepções de um serviço estejam vinculadas a uma avaliação subjetiva, é imprescindível que o profissional conheça as expectativas dos usuários para trazer melhorias no desempenho do trabalho e para isso é necessário ouvi-los, uma vez que os usuários dependem dos serviços e possuem a habilidade de julgar a qualidade percebida dos serviços de saúde e sugerir ideias úteis de melhoria ${ }^{4,42}$.

O serviço de saúde pode ser afetado pelo grau de percepção dos profissionais, assim a satisfação de suas necessidades no trabalho é essencial para a melhoria da produtividade e está atrelada à motivação. Dessa forma, para que o trabalho represente fonte de satisfação, é preciso que as relações estabelecidas sejam satisfatórias para o profíssional e para o usuário ${ }^{2}$.

\section{Considerações finais}

As pesquisas sobre a qualidade dos serviços públicos de saúde no Brasil, no período avaliado, enfocaram, principalmente, a percepção dos usuários.

$\mathrm{Na}$ atenção básica, algumas características de qualidade foram comuns aos usuários e aos profissionais: boa relação profissional-usuário, número adequado de profissionais e boas condições na es- 
trutura física. Outros itens foram descritos apenas pelos usuários: tempo de espera, cordialidade no atendimento e resolução dos problemas; e outro apenas pelos profissionais, a educação continuada. Analisando os últimos itens, percebe-se uma correlação entre as aspirações dos usuários e dos profissionais, pois a educação continuada pode solucionar os problemas relacionados a tempo de espera, a cordialidade no atendimento e a resolução dos problemas.

Os serviços de média complexidade apresentaram o item facilidade para agendar consulta comum à percepção dos usuários e dos profissionais. Os profissionais mencionaram outras características importantes para a qualidade: a organização do serviço e o trabalho em equipe, já os usuários citaram o tempo de espera, a cordialidade no atendimento, a resolução dos problemas e a boa relação profissional-usuário. Mais uma vez, nota-se uma correlação entre as percepções, ou seja, se o serviço de saúde estiver organizado e existir trabalho em equipe, o usuário poderá ser beneficiado com um menor tempo de espera, com um atendimento cordial, tendo seus problemas de saúde resolvidos e estabelecendo-se uma boa relação entre usuários e profissionais.

A boa relação profissional-usuário é comum à percepção dos usuários e aos profissionais nos serviços de alta complexidade. O tempo de espera, as boas condições na estrutura física, a capacitação profissional, a cordialidade no atendimento são características de qualidade mencionadas pelos usuários dos serviços de saúde e que se correlacionam com o desejo dos profissionais, com o trabalho em equipe e a motivação profissional.

\section{Agradecimentos}

À Fundação de Amparo à Pesquisa do Estado de São Paulo (Fapesp), por conceder bolsa de estudo que viabilizou a realização deste estudo.

\section{Abstract}

The SUS has assured the universalization of the right to health services efficient, making the search for quality a permanent challenge. In this challenge, the perception of users and professionals is important to assess the quality of health services, in primary care, secondary or tertiary. Objective: The objective was to verify evidence capable of sustaining the limitations of research on quality in the public health services, through a systematic discussion. Materials and method: It was performed a search in bibliographic databases Medline, Lilacs and Scielo, in the period from 1990 to 2013, using the keywords: quality in health services, user satisfaction, consumer satisfaction, job satisfaction and health workers. Results: The results showed that the primary attention has been object of analysis application in most studies, the Southeast region predominated as local search, the method of quantitative research was the most used and the perception of the users, the more measured. Conclusion: It was concluded that in primary care, the good professional relationship-user, adequate number of professionals and maintenance of the site were characteristics of quality common to users and professionals. The secondary care presented the item easy to schedule consultation common perception of users and professionals. The good professional relationship-user was the common perception of users and professionals in tertiary care.

Keywords: Quality of Health Care. Epidemiology. Unified Health System.

\section{Referências}

1. Martha SN; Gatti MAN; Vitta A; Simeão SFAP; Conti MHS; Saes SO, et al. Programa saúde da família sob a visão do usuário. Salusvita 2011; 30(3):159-77.

2. Volpato LF; Meneghim MC; Pereira AC; Ambrosano GMB. Planejamento da qualidade nas unidades de saúde da família, utilizando o Desdobramento da Função Qualidade (QFD). Cad Saúde Pública 2010; 26(8):1561-72.

3. Roncalli AG. O desenvolvimento das políticas públicas de saúde no Brasil e a construção do Sistema Único de Saúde. In: Pereira AC (org.). Odontologia em saúde coletiva: planejando ações e promovendo saúde. Porto Alegre Artmed; 2003. p. 28-49.

4. Fadel MAV; Regis Filho GI. Percepção da qualidade em serviços públicos de saúde: um estudo de caso. Rev Adm Pública 2009; 43(1):7-22.

5. Polizer R; D'Innocenzo M. Satisfação do cliente na avaliação da assistência de enfermagem. Rev Bras Enferm 2006: 59(4):548-51

6. Samico I; Hartz ZMA; Felisberto E; Carvalho EF. Atenção à saúde da criança: uma análise do grau de implantação e da satisfação de profissionais e usuários em dois municípios do estado de Pernambuco, Brasil. Rev Bras Saude Mater Infant 2005; 5(2): 229-40.

7. Dias OV; Vieira MA; Dias JP; Ramos LH. As dimensões da satisfação dos usuários do Programa Saúde da Família: confiabilidade e empatia. Acta Paul Enferm 2011; 24(2): 225-31.

8. Ribeiro JM; Siqueira SAV; Pinto LFS. Avaliação da atenção à saúde da criança (0-5 anos) no PSF de Teresópolis (RJ) segundo a percepção dos usuários. Ciênc Saúde Coletiva 2010; 15(2): 517-27.

9. Vaitsman J; Andrade GRB. Satisfação e responsividade: formas de medir a qualidade e a humanização da assistência à saúde. Ciênc Saúde Coletiva 2005; 10(3):599-613

10. Bittencourt RJ; Hortale VA. A qualidade nos serviços de emergência de hospitais públicos e algumas considerações sobre a conjuntura recente no município do Rio de Janeiro. Ciênc Saúde Coletiva 2007; 12(4):929-34.

11. Oliveira EM; Sipiri WC. Programa Saúde da Família: a experiência de equipe multiprofissional. Rev Saúde Pública 2006; 40(4):727-33.

12. Rocha ESB; Trevizan MA. Gerenciamento da qualidade em um serviço de enfermagem hospitalar. Rev Latinoam Enfermagem 2009; 17(2):online.

13. Assunção MCF; Santos IS; Gigante DP. Atenção primária em diabetes no sul do Brasil: estrutura, processo e resultados. Rev Saúde Pública 2001; 35(1):88-95.

14. Kotaka F; Pacheco MLR; Higaki Y. Avaliação pelos usuários dos hospitais participantes do programa de qualidade hospitalar no Estado de São Paulo, Brasil. Rev Saúde Pública 1997; 31(2):171-7. 
15. Ramos DD; Lima MADS. Acesso e acolhimento aos usuários em uma unidade de saúde de Porto Alegre, Rio Grande de Sul, Brasil. Cad Saúde Pública 2003; 19(1): 27-34.

16. Ronzani TM, Silva CM. O Programa Saúde da Família segundo profissionais de saúde, gestores e usuários. Ciênc Saúde Coletiva 2008; 13(1):23-34.

17. Halal IS; Sparrenberger F; Bertoni AM; Ciacomet C; Seibel CE; Lahude FM, et al. Avaliação da qualidade de assistência primária à saúde em localidade urbana da região sul do Brasil. Rev Saúde Pública 1994; 28(2):131-6.

18. Franco SC, Campos GWS. Avaliação da qualidade de atendimento ambulatorial em pediatria em um hospital universitário. Cad Saúde Pública 1998; 14(1):61-70.

19. Kloetzel K; Bertoni AM; Irazoqui MC; Campos VPG; Santos RN. Controle de qualidade em atenção primária à saúde. A satisfação do usuário. Cad Saúde Pública 1998; 14(3):623-8.

20. Kovacs MH; Feliciano VO; Sarinho SW; Veras AACA. Acessibilidade às ações básicas entre crianças atendidas em serviços de pronto-socorro. J Pediatr 2005; 81(3): 251-8.

21. Silveira JAM; Hayashi L; Scarpi MJ. Identificação de necessidades e expectativas dos pacientes de uma clínica de catarata. Arq Bras Oftalmol 2005; 68(5): 639-44.

22. Ferri SMN; Pereira MJB; Mishima SM; Caccia-Bava MCG; Almeida MCP. As tecnologias leves como geradoras de satisfação em usuários de uma unidade de saúde da família. Interface Comun Saúde Educ 2007; 11(23):515-29.

23. Jorge MSB; Guimarães JMX; Vieira LB; Paiva FDS; Silva DR; Pinto AGA. Avaliação da qualidade do Programa Saúde da Família no Ceará: a satisfação dos usuários. ver. Baiana Saúde Pública 2007; 31(2): 256-66.

24. Lima MAD; Ramos DD; Rosa RB; Nauderer TM; Davis R. Acesso e acolhimento em unidades de saúde na visão dos usuários. Acta Paul Enferm 2007; 20(1): 12-7.

25. Paiva SMA; Gomes ELR. Hospital care: assessment of users' satisfaction during hospital stay. Rev Latinoam Enfermagem 2007; 15(5):973-9

26. Santos SAS; Meneghim MC; Pereira AC. Análise da organização da demanda e grau de satisfação do profissional e usuário nas unidades de serviço público odontológico do $\mathrm{Mu}$ nicípio de Campos dos Goyatacazes/RJ/Brasil. Rev Odontol Unesp 2007; 36(2):169-74.

27. De Marco PF; Cítero VA; Moraes E; Nogueira-Martins LA. O impacto do trabalho em saúde mental: transtornos psiquiátricos menores, qualidade de vida e satisfação profissional. J Bras Psiquiatr 2008; 57(3):178-83.

28. Moura ERF; Santos MS; Pereira CG; Rocha CP. Santos LKX. A voz do usuário e a gerência do serviço de saúde. Rev APS $2008 ; 11(2): 181-8$.

29. Mendes ACG; Araújo Júnior JLCA; Furtado BMAS; Duarte PO; Santiago RF; Costa TR. Avaliação da satisfação dos usuários com a qualidade do atendimento nas grandes emergências do Recife, Pernambuco, Brasil. Rev Bras Saude Matern Infant 2009; 9(2):157-65.

30. Souza ACC; Moreira TMM; Silva MRF; Almeida PC. Acesso ao serviço de emergência pelos usuários com crise hipertensiva em um hospital de Fortaleza, CE, Brasil. Rev Bras Enferm 2009; 62(4):535-9.

31. Benazzi LEB; Figueiredo ACL; Bassani DG. Avaliação do usuário sobre o atendimento oftalmológico oferecido pelo SUS em um centro urbano no sul do Brasil. Ciênc Saúde Coletiva $2010 ; 15(3): 861-8$.

32. Lima ACS; Cabral ED; Vasconcelos MMVB. Satisfação dos usuários assistidos nos Centros de Especialidades Odontoló- gicas do município do Recife, Pernambuco, Brasil. Cad Saúde Pública 2010; 26(5):991-1002.

33. Medeiros FA; Souza GCA; Barbosa AAA; Costa ICC. Acolhimento em uma Unidade Básica de Saúde: a satisfação do usuário em foco. Rev Salud Publica 2010; 12(3):402-13.

34. Nery NG; Marcelo VC; Dantas MAA. A satisfação de idosos quanto à estratégia saúde da família, a partir da atenção em saúde bucal. Rev Bras Ciênc Saúde 2010; 14(1):43-500

35. Schwartz TD; Ferreira TB; Maciel ELN; Lima RCD. Estratégia saúde da família: avaliando o acesso ao SUS a partir da percepção dos usuários da unidade de saúde de resistência, na região de São Pedro, no município de Vitória/ES. Ciênc Saúde Coletiva 2010; 15(4):43-50

36. Souza RCR; Soares E. Perspectivas dos enfermeiros no prisma das demandas dos usuários em ouvidoria. Rev Enferm UERJ 2010; 18(3):411-6.

37. Gonçalves JR; Veras FEL; Matos ACM; Lima ISA. Avaliação da satisfação dos pacientes submetidos à intervenção fisioterapêutica no município de Campo Maior, PI. Fisioter mov 2011; 24(1):47-56

38. Ricci NA; Wanderly FS; Oliveira MS; Rebelatto JR. O hospital-escola de São Carlos: análise do funcionamento por meio da satisfação dos usuários. Ciênc Saúde Coletiva 2011; 16(supl.1): 1125-34.

39. Rosa RB; Pelegrini AHW; Lima MADS. Resolutividade da assistência e satisfação de usuários da Estratégia Saúde da Família. Rev Gaúcha Enferm 2011; 32(2): 345-51.

40. Serapioni M; Silva MGC. Avaliação da qualidade do programa Saúde da Família em municípios do Ceará: uma abordagem multidimensional. Ciênc Saúde Coletiva 2011; 16 (11).207-27

41. Pena MM; Melleiro MM. Grau de satisfação de usuários de um hospital privado. Acta Paul Enferm 2012; 25(2):197-203.

42. Sousa AA; Coleta MFD. O bem-estar no trabalho de psicólogos em serviços de saúde pública. Psicol Cienc Prof 2012; 32(2):404-21.

43. Santiago RF; Mendes ACG; Miranda GMD; Duarte PO; Furtado BMASM; Souza WS. Qualidade do atendimento nas Unidades de Saúde da Família no município de Recife: a percepção dos usuários. Ciênc Saúde Coletiva. 2013; 18(1):4315-26.

44. Tanaka OY; Melo CM. Reflexões sobre a Avaliação em Serviços de Saúde e a Adoção das Abordagens Qualitativa e Quantitativa. In: Maria Lúcia Magalhães Bosi; Francisco Javier Mercado. (Org.). Pesquisa Qualitativa de Serviços de Saúde. São Paulo: Editora Vozes; 2004. p. 121-36.

45. Serapioni M. Métodos qualitativos e quantitativos na pesquisa social em saúde: algumas estratégias para a integração. Ciênc Saúde Coletiva 2000; 5(1):187-92.

46. Minayo MCS; Sanches O. Quantitativo-qualitativo: oposição ou complementaridade? Cad Saúde Pública 1993; 9(3): 239-62.

47. Esperidião MA; Trad LAB. Avaliação de satisfação de usuários: considerações teórico-conceituais. Cad Saúde Pública 2006; 22(6):1267-76.

48. Damasceno RF; Souza LPS; Ruas MFL; Brito PA; Silva EA; Silva JLS. O acolhimento no contexto da Estratégia Saúde da Família. J Health Sci Inst 2012; 30(1): 37-40.

49. Jorge MSB; Guimarães JMX; Vieira LB; Paiva FDS; Silva DR; Pinto AGA. Avaliação da qualidade do Programa Saúde da Família no Ceará: a satisfação dos usuários. Rev Baiana Saude Publica. 2007; 31(2):256-66. 
50. Novaes MRCG; Lima ACV; Nogueira DY; Cerqueira F; Mello GF; Souza HS, et al. Estudo etnográfico e de satisfação de profissionais e usuários do Programa de Saúde da Família em Samambaia, Distrito Federal. Comun Ciênc Saúde 2010; 21(4):289-300.

51. Oliveira SF; Albuquerque FJB. Programa de saúde da família: uma análise a partir das crenças dos seus prestadores de serviço. Psicol Soc 2008; 20(2):237-46.

\section{Endereço para correspondência:}

Luciana Fernandes Volpato

Avenida Limeira, 901 Caixa Postal 52

13414-903 Piracicaba - SP

Fone: (19) 2106-5209

E-mail: lucianavopato@superig.com.br

Recebido: 12/10/2013. Aceito: 06/08/2014. 